\title{
An efficient strategy for the synthesis of 1-chloroethyl phosphates and phosphoramidates
}

\author{
Hanna Kumpulainen ${ }^{* \dagger}$, Tomi Järvinen ${ }^{\dagger}$, Raimo Saari ${ }^{\dagger}$, Marko Lehtonen $^{\dagger}$, Jouko Vepsäläinen ${ }^{*}$
}

Department of Pharmaceutical Chemistry and Department of Chemistry, University of Kuopio, P.O. Box 1627, FI-70211 Kuopio, Finland.

Hanna.Kumpulainen@uku.fi

\section{Supporting Information}

$\begin{array}{lll}\text { CONTENTS Page } & \text { Pag }\end{array}$

1. General methods ............................................... 2

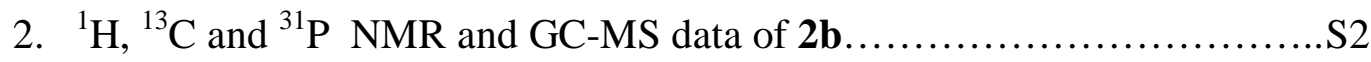

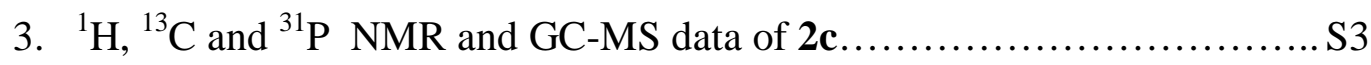

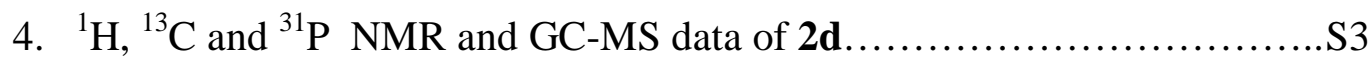

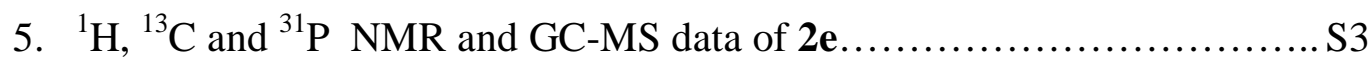

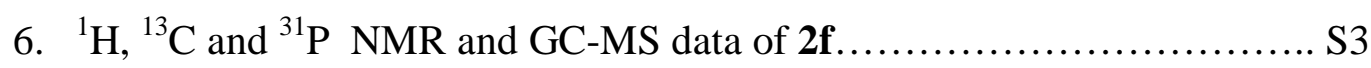

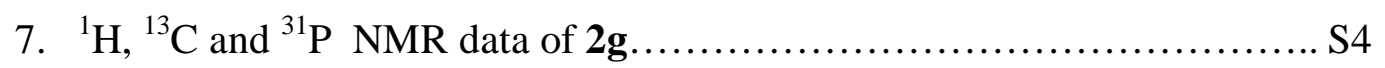

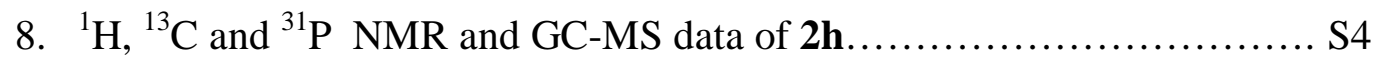

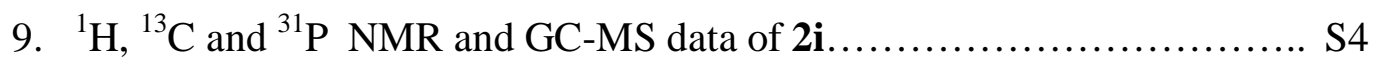


10. ${ }^{1} \mathrm{H},{ }^{13} \mathrm{C}$ and ${ }^{31} \mathrm{P}$ NMR, GC-MS and CHNS data of $\mathbf{3 b} \ldots \ldots \ldots \ldots \ldots \ldots . . . . . . . . . . .4$

11. Figure 1. As an example of the complicate structure of the spectra the......S5 calculated and observed ${ }^{1} \mathrm{H}$ NMR spectrum for $\mathrm{OCH}_{2}$-protons signals of $\mathbf{3 b}^{24}$

12. ${ }^{1} \mathrm{H},{ }^{13} \mathrm{C}$ and ${ }^{31} \mathrm{P}$ NMR, GC-MS and CHNS data of $3 \mathbf{c} \ldots \ldots \ldots \ldots \ldots \ldots \ldots . . . . . . . . . .55$

13. ${ }^{1} \mathrm{H},{ }^{13} \mathrm{C}$ and ${ }^{31} \mathrm{P}$ NMR, GC-MS and CHNS data of 3d....................S6

14. ${ }^{1} \mathrm{H},{ }^{13} \mathrm{C}$ and ${ }^{31} \mathrm{P}$ NMR, GC-MS and CHNS data of 3e....................S6

15. ${ }^{1} \mathrm{H},{ }^{13} \mathrm{C}$ and ${ }^{31} \mathrm{P}$ NMR, GC-MS and CHNS data of $\mathbf{3 f} \ldots \ldots \ldots \ldots \ldots \ldots \ldots . . . . . . . . .6$

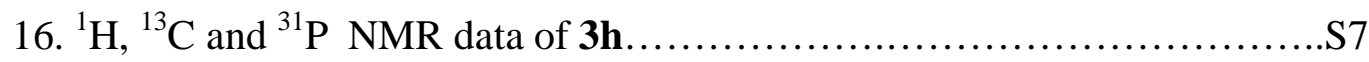

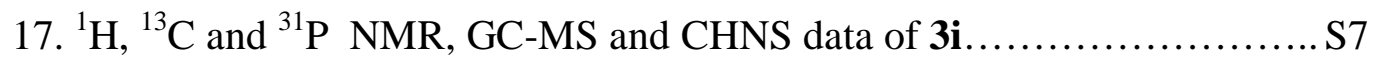

General methods. All the described reactions were performed under argon atmosphere. Column chromatography was performed with silica gel (0.063-0.200 mm mesh). ${ }^{1} \mathrm{H},{ }^{13} \mathrm{C}$ and ${ }^{31} \mathrm{P}$ NMR-spectra were recorded at $500.13,125.78$ and $200.46 \mathrm{MHz}$ at $25^{\circ} \mathrm{C}$, respectively. TMS was used as an internal reference for ${ }^{1} \mathrm{H}$ and ${ }^{13} \mathrm{C}$ measurements and $\mathrm{H}_{3} \mathrm{PO}_{4}$ as an external reference for ${ }^{31} \mathrm{P}$ measurements. The splitting pattern abbreviations are as follows: $\mathrm{s}=$ singlet, $\mathrm{d}=$ doublet, $\mathrm{t}=$ triplet, $\mathrm{q}=$ quartet, $\mathrm{m}=$ multiplet. The ${ }^{1} \mathrm{H}$ NMR parameters have been solved precisely with the PERCHit iterator ${ }^{23}$ under PERCH software. ${ }^{24}$ Electron impact or positive chemical ionization mass spectra were acquired by an gas chromatograph- mass spectrometry system. All reagents obtained from commercial suppliers were used without further purifications. Most of the products were oily compounds and difficult to obtain as analytically pure products. However, the spectral and GC-MS data of the compounds were consistent with those of the proposed structures.

Phosphoric acid diethyl ester vinyl ester 2b: $64 \%$, colorless oil. Chromatography eluent EtOAc:petrol ether 1:3. ${ }^{1} \mathrm{H}$ NMR $\left(\mathrm{CDCl}_{3}\right) \delta 6.584\left(1 \mathrm{H}, \mathrm{ddd}, \mathrm{J}=13.54 \mathrm{~Hz}, 5.87 \mathrm{~Hz},{ }^{3} \mathrm{~J}_{\mathrm{HP}}=6.50 \mathrm{~Hz}\right), 4.901$ $\left(1 \mathrm{H}\right.$, ddd, J=13.54 Hz, $\left.2.05 \mathrm{~Hz},{ }^{4} \mathrm{~J}_{\mathrm{HP}}=1.19 \mathrm{~Hz}\right), 4.568\left(1 \mathrm{H}, \mathrm{ddd}, \mathrm{J}=5.87 \mathrm{~Hz}, 2.05 \mathrm{~Hz},{ }^{4} \mathrm{~J}_{\mathrm{HP}}=2.71 \mathrm{~Hz}\right)$, 
$4.174\left(4 \mathrm{H}, \mathrm{dq}, \mathrm{J}=7.08 \mathrm{~Hz},{ }^{3} \mathrm{~J}_{\mathrm{HP}}=8.28 \mathrm{~Hz}\right), 1.363\left(6 \mathrm{H}, \mathrm{td}, \mathrm{J}=7.08 \mathrm{~Hz},{ }^{3} \mathrm{~J}_{\mathrm{HP}}=1.01 \mathrm{~Hz}\right) ;{ }^{13} \mathrm{C}$ NMR $\left(\mathrm{CDCl}_{3}\right) \delta$ $142.17\left(\mathrm{~d},{ }^{2} \mathrm{~J}_{\mathrm{CP}}=5.6 \mathrm{~Hz}\right), 99.63\left(\mathrm{~d},{ }^{3} \mathrm{~J}_{\mathrm{CP}}=10.4 \mathrm{~Hz}\right), 64.33\left(\mathrm{~d},{ }^{2} \mathrm{~J}_{\mathrm{CP}}=5.8 \mathrm{~Hz}\right), 16.00\left(\mathrm{~d},{ }^{3} \mathrm{~J}_{\mathrm{CP}}=6.6 \mathrm{~Hz}\right)$; ${ }^{31} \mathrm{P}$ NMR $\left(\mathrm{CDCl}_{3}\right) \delta-4.28$. GC-MS $\mathrm{m} / z .180(\mathrm{M})$.

Phosphoric acid bis-(2,2,2-trichloro-ethyl) ester vinyl ester 2c: $68 \%$, colorless oil. Chromatography eluent EtOAc:petrol ether 1:2. ${ }^{1} \mathrm{H}$ NMR $\left(\mathrm{CDCl}_{3}\right) \delta 6.630(1 \mathrm{H}$, ddd, J=13.44 Hz, 5.78 $\left.\mathrm{Hz},{ }^{3} \mathrm{~J}_{\mathrm{HP}}=6.71 \mathrm{~Hz}\right), 5.082\left(1 \mathrm{H}, \mathrm{ddd}, \mathrm{J}=13.44 \mathrm{~Hz}, 2.59 \mathrm{~Hz},{ }^{4} \mathrm{~J}_{\mathrm{HP}}=1.30 \mathrm{~Hz}\right), 4.758(1 \mathrm{H}, \mathrm{ddd}, \mathrm{J}=5.78 \mathrm{~Hz}$, $\left.2.59 \mathrm{~Hz},{ }^{4} \mathrm{~J}_{\mathrm{HP}}=2.66 \mathrm{~Hz}\right), 4.680\left(4 \mathrm{H}, \mathrm{d},{ }^{3} \mathrm{~J}_{\mathrm{HP}}=6.90 \mathrm{~Hz}\right) ;{ }^{13} \mathrm{C} \mathrm{NMR}\left(\mathrm{CDCl}_{3}\right) \delta 141.34\left(\mathrm{~d},{ }^{2} \mathrm{~J}_{\mathrm{CP}}=6.2 \mathrm{~Hz}\right)$, $102.17\left(\mathrm{~d},{ }^{3} \mathrm{~J}_{\mathrm{CP}}=10.6 \mathrm{~Hz}\right), 94.34\left(\mathrm{~d},{ }^{3} \mathrm{~J}_{\mathrm{CP}}=10.6 \mathrm{~Hz}\right), 77.42\left(\mathrm{~d},{ }^{2} \mathrm{~J}_{\mathrm{CP}}=4.5 \mathrm{~Hz}\right) ;{ }^{31} \mathrm{P} \mathrm{NMR}\left(\mathrm{CDCl}_{3}\right) \delta-$ 7.59. GC-MS $m / z 387(\mathrm{M})$.

Phosphoric acid methyl ester phenyl ester vinyl ester 2d: $54 \%$, colorless oil. Chromatography eluent EtOAc:petrol ether 1:4. ${ }^{1} \mathrm{H}$ NMR $\left(\mathrm{CDCl}_{3}\right) \delta 7.346\left(2 \mathrm{H}, \mathrm{m}, \mathrm{J}=8.05 \mathrm{~Hz}, 7.40 \mathrm{~Hz},{ }^{4} \mathrm{~J}_{\mathrm{HH}}=-1.74 \mathrm{~Hz}\right.$, $\left.{ }^{5} \mathrm{~J}_{\mathrm{HH}}=0.77 \mathrm{~Hz}\right), 7.218\left(2 \mathrm{H}, \mathrm{m}, \mathrm{J}=8.05 \mathrm{~Hz},{ }^{4} \mathrm{~J}_{\mathrm{HH}}=-1.20 \mathrm{~Hz},-1.17 \mathrm{~Hz},{ }^{5} \mathrm{~J}_{\mathrm{HH}}=0.77 \mathrm{~Hz},{ }^{4} \mathrm{~J}_{\mathrm{HP}}=1.19 \mathrm{~Hz}\right)$, $7.195\left(1 \mathrm{H}, \mathrm{m}, \mathrm{J}=7.40,{ }^{4} \mathrm{~J}_{\mathrm{HH}}=-1.17\right), 6.634\left(1 \mathrm{H}, \mathrm{ddd}, \mathrm{J}=13.50 \mathrm{~Hz}, 5.82 \mathrm{~Hz}\right.$ and $\left.{ }^{3} \mathrm{~J}_{\mathrm{HP}}=6.59 \mathrm{~Hz}\right), 4.966(1 \mathrm{H}$, ddd, $\mathrm{J}=13.50 \mathrm{~Hz}, 2.27 \mathrm{~Hz}$ and $\left.{ }^{4} \mathrm{~J}_{\mathrm{HP}}=1.19 \mathrm{~Hz}\right), 4.825\left(1 \mathrm{H}, \mathrm{ddd}, \mathrm{J}=5.82 \mathrm{~Hz}, 2.27 \mathrm{~Hz}\right.$ and $\left.{ }^{4} \mathrm{~J}_{\mathrm{HP}}=2.80 \mathrm{~Hz}\right)$, $3.908\left(3 \mathrm{H}, \mathrm{d},{ }^{3} \mathrm{~J}_{\mathrm{HP}}=11.51 \mathrm{~Hz}\right) ;{ }^{13} \mathrm{C} \mathrm{NMR}\left(\mathrm{CDCl}_{3}\right) \delta 150.36\left(\mathrm{~d},{ }^{2} \mathrm{~J}_{\mathrm{CP}}=6.9 \mathrm{~Hz}\right), 141.99\left(\mathrm{~d},{ }^{2} \mathrm{~J}_{\mathrm{CP}}=5.9 \mathrm{~Hz}\right)$, $129.85,125.47,120.01\left(\mathrm{~d},{ }^{3} \mathrm{~J}_{\mathrm{CP}}=4.8 \mathrm{~Hz}\right), 100.70\left(\mathrm{~d},{ }^{3} \mathrm{~J}_{\mathrm{CP}}=10.5 \mathrm{~Hz}\right), 55.23\left(\mathrm{~d},{ }^{2} \mathrm{~J}_{\mathrm{CP}}=6.2 \mathrm{~Hz}\right) ;{ }^{31} \mathrm{P} \mathrm{NMR}$ $\left(\mathrm{CDCl}_{3}\right) \delta$-8.79. GC-MS $m / z 215(\mathrm{M}+1)$.

Phosphoric acid bis-(2,6-dimethyl-phenyl) ester vinyl ester 2e: $35 \%$, colorless oil. Chromatography eluent EtOAc:petrol ether 1:20. ${ }^{1} \mathrm{H}$ NMR $\left(\mathrm{CDCl}_{3}\right) \delta$ 7.044-6.977 $(6 \mathrm{H}, \mathrm{m}), 6.661(1 \mathrm{H}$, ddd, J=13.49 Hz, $5.77 \mathrm{~Hz}$ and $\left.{ }^{3} \mathrm{~J}_{\mathrm{HP}}=5.78 \mathrm{~Hz}\right), 4.840\left(1 \mathrm{H}, \mathrm{ddd}, \mathrm{J}=13.49 \mathrm{~Hz}, 2.19 \mathrm{~Hz}\right.$ and $\left.{ }^{4} \mathrm{~J}_{\mathrm{HP}}=1.01 \mathrm{~Hz}\right)$, $4.525\left(1 \mathrm{H}\right.$, ddd, J=5.77 Hz, $2.19 \mathrm{~Hz}$ and $\left.{ }^{4} \mathrm{~J}_{\mathrm{HP}}=3.91 \mathrm{~Hz}\right), 2.343(\mathrm{~s}, 12 \mathrm{H}) ;{ }^{13} \mathrm{C} \mathrm{NMR}\left(\mathrm{CDCl}_{3}\right) \delta 147.99(\mathrm{~d}$, $\left.{ }^{2} \mathrm{~J}_{\mathrm{CP}}=8.6 \mathrm{~Hz}\right), 142.45\left(\mathrm{~d},{ }^{2} \mathrm{~J}_{\mathrm{CP}}=5.4 \mathrm{~Hz}\right), 130.29\left(\mathrm{~d},{ }^{3} \mathrm{~J}_{\mathrm{CP}}=3.4 \mathrm{~Hz}\right), 129.16\left(\mathrm{~d},{ }^{4} \mathrm{~J}_{\mathrm{CP}}=1.5 \mathrm{~Hz}\right), 125.54(\mathrm{~d}$, $\left.{ }^{5} \mathrm{~J}_{\mathrm{CP}}=1.7 \mathrm{~Hz}\right), 99.57\left(\mathrm{~d},{ }^{3} \mathrm{~J}_{\mathrm{CP}}=11.3 \mathrm{~Hz}\right), 17.00 ;{ }^{31} \mathrm{P} \mathrm{NMR}\left(\mathrm{CDCl}_{3}\right) \delta-14.69$. GC-MS $\mathrm{m} / z 333(\mathrm{M}+1)$.

2-vinyloxy-[1,3,2]dioxaphosphinane 2-oxide 2f: $76 \%$, colorless oil. Chromatography eluent EtOAc:petrol ether 1:1. ${ }^{1} \mathrm{H}$ NMR $\left(\mathrm{CDCl}_{3}\right) \delta 6.611\left(1 \mathrm{H}\right.$, ddd, J=13.56 Hz, $5.87 \mathrm{~Hz}$ and $\left.{ }^{3} \mathrm{~J}_{\mathrm{HP}}=7.47 \mathrm{~Hz}\right)$, 
$4.962\left(1 \mathrm{H}\right.$, ddd, $\mathrm{J}=13.56 \mathrm{~Hz}, 2.28 \mathrm{~Hz}$ and $\left.{ }^{4} \mathrm{~J}_{\mathrm{HP}}=1.21 \mathrm{~Hz}\right), 4.637(1 \mathrm{H}$, ddd, $\mathrm{J}=5.87 \mathrm{~Hz}, 2.28 \mathrm{~Hz}$ and $\left.{ }^{4} \mathrm{~J}_{\mathrm{HP}}=2.12 \mathrm{~Hz}\right), 4.520-4.400(4 \mathrm{H}, \mathrm{m}), 2.390-2.287(1 \mathrm{H}, \mathrm{m}), 1.834-1.779(1 \mathrm{H}, \mathrm{m}) ;{ }^{13} \mathrm{C} \mathrm{NMR}\left(\mathrm{CDCl}_{3}\right) \delta$ $141.64\left(\mathrm{~d},{ }^{2} \mathrm{~J}_{\mathrm{CP}}=5.7 \mathrm{~Hz}\right), 100.22\left(\mathrm{~d},{ }^{3} \mathrm{~J}_{\mathrm{CP}}=9.9 \mathrm{~Hz}\right), 69.23\left(\mathrm{~d},{ }^{2} \mathrm{~J}_{\mathrm{CP}}=6.3 \mathrm{~Hz}\right), 25.42\left(\mathrm{~d},{ }^{3} \mathrm{~J}_{\mathrm{CP}}=7.3 \mathrm{~Hz}\right)$; ${ }^{31} \mathrm{P}$ NMR $\left(\mathrm{CDCl}_{3}\right) \delta-11.15 . \mathrm{GC}-\mathrm{MS} \mathrm{m} / z 165(\mathrm{M}+1)$.

Phosphoric acid diphenyl ester vinyl ester 2g: $70 \%$, light-yellow oil. Chromatography eluent EtOAc:petrol ether 1:4. ${ }^{1} \mathrm{H}$ NMR $\left(\mathrm{CDCl}_{3}\right) \delta 7.451\left(4 \mathrm{H}, \mathrm{m}, \mathrm{J}=8.20 \mathrm{~Hz}, 7.44 \mathrm{~Hz},{ }^{4} \mathrm{~J}_{\mathrm{HH}}=-2.35 \mathrm{~Hz},{ }^{5} \mathrm{~J}_{\mathrm{HH}}=\right.$ $0.55 \mathrm{~Hz}), 7.290\left(2 \mathrm{H}, \mathrm{m}, \mathrm{J}=7.44,{ }^{4} \mathrm{~J}_{\mathrm{HH}}=-1.04\right), 7.265\left(4 \mathrm{H}, \mathrm{m}, \mathrm{J}=8.20 \mathrm{~Hz},{ }^{4} \mathrm{~J}_{\mathrm{HH}}=-2.06 \mathrm{~Hz},-1.04 \mathrm{~Hz},{ }^{5} \mathrm{~J}_{\mathrm{HH}}=\right.$ $\left.0.55 \mathrm{~Hz},{ }^{4} \mathrm{~J}_{\mathrm{HP}}=1.22 \mathrm{~Hz}\right), 6.845\left(1 \mathrm{H}, \mathrm{ddd}, \mathrm{J}=13.34 \mathrm{~Hz}, 5.76 \mathrm{~Hz}\right.$ and $\left.{ }^{3} \mathrm{~J}_{\mathrm{HP}}=6.82 \mathrm{~Hz}\right), 5.056(1 \mathrm{H}, \mathrm{ddd}$, $\mathrm{J}=13.34 \mathrm{~Hz}, 2.38 \mathrm{~Hz}$ and $\left.{ }^{4} \mathrm{~J}_{\mathrm{HP}}=1.23 \mathrm{~Hz}\right), 4.825\left(1 \mathrm{H}, \mathrm{ddd}, \mathrm{J}=5.76 \mathrm{~Hz}, 2.38 \mathrm{~Hz}\right.$ and $\left.{ }^{4} \mathrm{~J}_{\mathrm{HP}}=2.79 \mathrm{~Hz}\right)$.

Phosphoric acid dibenzyl ester vinyl ester 2h: $30 \%$, colorless oil. Chromatography eluent EtOAc:petrol ether 1:4. ${ }^{1} \mathrm{H}$ NMR $\left(\mathrm{CDCl}_{3}\right) \delta$ 7.386-7.314 $(12 \mathrm{H}, \mathrm{m}), 6.529(1 \mathrm{H}, \mathrm{ddd}, \mathrm{J}=13.51 \mathrm{~Hz}, 5.85$ $\mathrm{Hz}$ and $\left.{ }^{3} \mathrm{~J}_{\mathrm{HP}}=6.51 \mathrm{~Hz}\right), 5.083\left(4 \mathrm{H}, \mathrm{d},{ }^{3} \mathrm{~J}_{\mathrm{HP}}=8.23\right), 4.862\left(1 \mathrm{H}\right.$, ddd, $\mathrm{J}=13.51 \mathrm{~Hz}, 2.16 \mathrm{~Hz}$ and ${ }^{4} \mathrm{~J}_{\mathrm{HP}}=1.23$ $\mathrm{Hz}), 4.544\left(1 \mathrm{H}, \mathrm{ddd}, \mathrm{J}=5.85 \mathrm{~Hz}, 2.16 \mathrm{~Hz}\right.$ and $\left.{ }^{4} \mathrm{~J}_{\mathrm{HP}}=2.70 \mathrm{~Hz}\right) ;{ }^{13} \mathrm{C} \mathrm{NMR}\left(\mathrm{CDCl}_{3}\right) \delta 141.96\left(\mathrm{~d},{ }^{2} \mathrm{~J}_{\mathrm{CP}}=5.9\right.$ $\mathrm{Hz}), 135.40\left(\mathrm{~d},{ }^{3} \mathrm{~J}_{\mathrm{CP}}=6.9 \mathrm{~Hz}\right), 128.69,128.62,128.04,100.23\left(\mathrm{~d},{ }^{3} \mathrm{~J}_{\mathrm{CP}}=10.4 \mathrm{~Hz}\right), 69.77\left(\mathrm{~d},{ }^{2} \mathrm{~J}_{\mathrm{CP}}=5.6\right.$ $\mathrm{Hz}) ;{ }^{31} \mathrm{P} \mathrm{NMR}\left(\mathrm{CDCl}_{3}\right) \delta$ 3.93. GC-MS $m / z 305(\mathrm{M}+1)$.

N,N-diethylphosphoramidic acid methyl ester vinyl ester $2 \mathbf{i}: 88 \%$, colorless oil. Chromatography eluent EtOAc:petrol ether 1:1. ${ }^{1} \mathrm{H}$ NMR $\left(\mathrm{CDCl}_{3}\right) \delta 6.576\left(1 \mathrm{H}\right.$, ddd, $\mathrm{J}=13.60 \mathrm{~Hz}, 5.93 \mathrm{~Hz}$ and ${ }^{3} \mathrm{~J}_{\mathrm{HP}}=6.73$ $\mathrm{Hz}), 4.827\left(1 \mathrm{H}\right.$, ddd, $\mathrm{J}=13.60 \mathrm{~Hz}, 1.76 \mathrm{~Hz}$ and $\left.{ }^{4} \mathrm{~J}_{\mathrm{HP}}=1.33 \mathrm{~Hz}\right), 4.505(1 \mathrm{H}$, ddd, J=5.93 Hz, $1.76 \mathrm{~Hz}$ and $\left.{ }^{4} \mathrm{~J}_{\mathrm{HP}}=2.21 \mathrm{~Hz}\right), 3.722\left(3 \mathrm{H}, \mathrm{d},{ }^{3} \mathrm{~J}_{\mathrm{HP}}=11.32 \mathrm{~Hz}\right), 3.104\left(4 \mathrm{H}, \mathrm{dq}, \mathrm{J}=7.11 \mathrm{~Hz},{ }^{3} \mathrm{~J}_{\mathrm{HP}}=11.87 \mathrm{~Hz}\right), 1.122(6 \mathrm{H}, \mathrm{t}$, $\mathrm{J}=7.11 \mathrm{~Hz} ;{ }^{13} \mathrm{C} \mathrm{NMR}\left(\mathrm{CDCl}_{3}\right) \delta 142.39\left(\mathrm{~d},{ }^{2} \mathrm{~J}_{\mathrm{CP}}=5.3 \mathrm{~Hz}\right), 98.88\left(\mathrm{~d},{ }^{3} \mathrm{~J}_{\mathrm{CP}}=10.2 \mathrm{~Hz}\right), 53.05\left(\mathrm{~d},{ }^{2} \mathrm{~J}_{\mathrm{CP}}=5.7\right.$ $\mathrm{Hz}), 39.79\left(\mathrm{~d},{ }^{2} \mathrm{~J}_{\mathrm{CP}}=4.5 \mathrm{~Hz}\right), 14.17\left(\mathrm{~d},{ }^{3} \mathrm{~J}_{\mathrm{CP}}=1.5 \mathrm{~Hz}\right) ;{ }^{31} \mathrm{P} \mathrm{NMR}\left(\mathrm{CDCl}_{3}\right) \delta-9.14 . \mathrm{GC}-\mathrm{MS} m / z 193(\mathrm{M})$.

Phosphoric acid 1-chloro-ethyl ester diethyl ester 3b: $91 \%$ (without purification), yellow viscose oil. Chromatography eluent hexane:EtOAc 1:1. ${ }^{1} \mathrm{H}$ NMR $\left(\mathrm{CDCl}_{3}\right) \delta 6.247(1 \mathrm{H}$, dq, J=5.61 $\mathrm{Hz}$, $\left.{ }^{3} \mathrm{~J}_{\mathrm{HP}}=7.79 \mathrm{~Hz}\right), 4.201 *\left(1 \mathrm{H}, \mathrm{ddq},{ }^{2} \mathrm{~J}_{\mathrm{HH}}=-10.07 \mathrm{~Hz}, \mathrm{~J}=7.10 \mathrm{~Hz},{ }^{3} \mathrm{~J}_{\mathrm{HP}}=7.79 \mathrm{~Hz}\right), 4.184 *\left(1 \mathrm{H}, \mathrm{ddq},{ }^{2} \mathrm{~J}_{\mathrm{HH}}=-\right.$ 
$\left.10.07 \mathrm{~Hz}, \mathrm{~J}=7.05 \mathrm{~Hz}{ }^{3} \mathrm{~J}_{\mathrm{HP}}=8.05 \mathrm{~Hz}\right), 4.159^{*}\left(1 \mathrm{H}, \mathrm{ddq},{ }^{2} \mathrm{~J}_{\mathrm{HH}}=-10.13 \mathrm{~Hz}, \mathrm{~J}=7.08 \mathrm{~Hz},{ }^{3} \mathrm{~J}_{\mathrm{HP}}=8.08 \mathrm{~Hz}\right)$, 4.136* $\left(1 \mathrm{H}, \mathrm{ddq},{ }^{2} \mathrm{~J}_{\mathrm{HH}}=-10.13 \mathrm{~Hz}, \mathrm{~J}=7.06 \mathrm{~Hz},{ }^{3} \mathrm{~J}_{\mathrm{HP}}=7.99 \mathrm{~Hz}\right), 1.845\left(3 \mathrm{H}, \mathrm{dd}, \mathrm{J}=5.61 \mathrm{~Hz},{ }^{4} \mathrm{~J}_{\mathrm{HP}}=1.03 \mathrm{~Hz}\right)$, $1.368\left(3 \mathrm{H}\right.$, ddd, J=7.10 Hz, $\left.7.05 \mathrm{~Hz},{ }^{4} \mathrm{~J}_{\mathrm{HP}}=1.13 \mathrm{~Hz}\right), 1.358\left(3 \mathrm{H}, \mathrm{ddd}, \mathrm{J}=7.08 \mathrm{~Hz}, 7.06 \mathrm{~Hz},{ }^{4} \mathrm{~J}_{\mathrm{HP}}=1.09 \mathrm{~Hz}\right)$; ${ }^{13} \mathrm{C} \operatorname{NMR}\left(\mathrm{CDCl}_{3}\right) \delta 85.72\left(\mathrm{~d},{ }^{2} \mathrm{~J}_{\mathrm{CP}}=6.3 \mathrm{~Hz}\right), 64.58^{*}\left(\mathrm{~d},{ }^{2} \mathrm{~J}_{\mathrm{CP}}=6.1 \mathrm{~Hz}\right), 64.33^{*}\left(\mathrm{~d},{ }^{2} \mathrm{~J}_{\mathrm{CP}}=5.8 \mathrm{~Hz}\right), 27.65$ $\left(\mathrm{d},{ }^{3} \mathrm{~J}_{\mathrm{CP}}=8.2 \mathrm{~Hz}\right), 16.02\left(\mathrm{~d},{ }^{3} \mathrm{~J}_{\mathrm{CP}}=6.9 \mathrm{~Hz}\right) ;{ }^{31} \mathrm{P} \mathrm{NMR}\left(\mathrm{CDCl}_{3}\right) \delta-3.11 . \mathrm{GC}-\mathrm{MS} m / z 217(\mathrm{M}+1), 181(\mathrm{M}$ - Cl). Anal. Calcd for $\mathrm{C}_{6} \mathrm{H}_{14} \mathrm{ClO}_{4} \mathrm{P} \cdot 0.6 \mathrm{HCl}$ C, 30.22; H, 6.17. Found: C, 29.98; H, 5.94.

*Due to chiral center and prochirality there is four ${ }^{1} \mathrm{H}$ and two ${ }^{13} \mathrm{C}$ chemical shifts for $\mathrm{OCH}_{2}$-groups
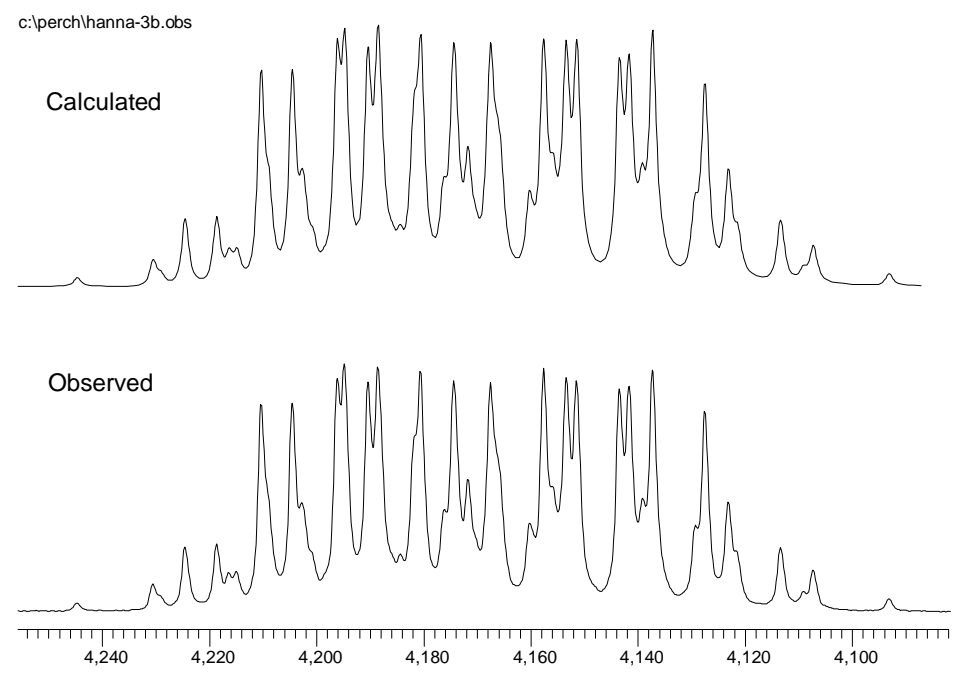

Figure 1. As an example of the complicate structure of the spectra the calculated and observed ${ }^{1} \mathrm{H}$ NMR spectrum for $\mathrm{OCH}_{2}$-protons signals of $\mathbf{3} \mathbf{b}^{24}$

Phosphoric acid 1-chloro-ethyl ester bis-(2,2,2-trichloro-ethyl) ester 3c: $66 \%$ (without purification), colorless viscose oil. Chromatography eluent $\mathrm{CH}_{2} \mathrm{Cl}_{2}$ :hexane:EtOAc (gradient). ${ }^{1} \mathrm{H} \mathrm{NMR}$ $\left(\mathrm{CDCl}_{3}\right){ }^{1} \mathrm{H} \mathrm{NMR}\left(\mathrm{CDCl}_{3}\right) \delta 6.332\left(1 \mathrm{H}, \mathrm{dq}, \mathrm{J}=5.63 \mathrm{~Hz},{ }^{3} \mathrm{~J}_{\mathrm{HP}}=7.40 \mathrm{~Hz}\right), 4.716^{*}\left(1 \mathrm{H}, \mathrm{dd},{ }^{2} \mathrm{~J}_{\mathrm{HH}}=-10.97 \mathrm{~Hz}\right.$, $\left.{ }^{3} \mathrm{~J}_{\mathrm{HP}}=6.82 \mathrm{~Hz}\right), 4.667 *\left(1 \mathrm{H}, \mathrm{dd},{ }^{2} \mathrm{~J}_{\mathrm{HH}}=-7.03 \mathrm{~Hz},{ }^{3} \mathrm{~J}_{\mathrm{HP}}=6.88 \mathrm{~Hz}\right), 4.666 *\left(1 \mathrm{H}, \mathrm{dd},{ }^{2} \mathrm{~J}_{\mathrm{HH}}=-10.97 \mathrm{~Hz}\right.$, $\left.{ }^{3} \mathrm{~J}_{\mathrm{HP}}=6.59 \mathrm{~Hz}\right), 4.661 *\left(1 \mathrm{H}, \mathrm{dd},{ }^{2} \mathrm{~J}_{\mathrm{HH}}=-7.03 \mathrm{~Hz},{ }^{3} \mathrm{~J}_{\mathrm{HP}}=6.44 \mathrm{~Hz}\right), 1.919\left(3 \mathrm{H}, \mathrm{dd}, \mathrm{J}=5.63 \mathrm{~Hz},{ }^{4} \mathrm{~J}_{\mathrm{HP}}=1.35\right.$ $\mathrm{Hz}) ;{ }^{13} \mathrm{C} \mathrm{NMR}\left(\mathrm{CDCl}_{3}\right) \delta 94.28,86.27\left(\mathrm{~d},{ }^{2} \mathrm{~J}_{\mathrm{CP}}=6.8 \mathrm{~Hz}\right), 77.44 *\left(\mathrm{~d},{ }^{2} \mathrm{~J}_{\mathrm{CP}}=4.4 \mathrm{~Hz}\right), 77.27 *\left(\mathrm{~d},{ }^{2} \mathrm{~J}_{\mathrm{CP}}=4.2\right.$ $\mathrm{Hz}), 27.35\left(\mathrm{~d},{ }^{3} \mathrm{~J}_{\mathrm{CP}}=8.8 \mathrm{~Hz}\right) ;{ }^{31} \mathrm{P}$ NMR $\left(\mathrm{CDCl}_{3}\right) \delta-6.18$. GC-MS m/z $423(\mathrm{M}), 389(\mathrm{M}-\mathrm{Cl})$. Anal. Calcd for $\mathrm{C}_{6} \mathrm{H}_{8} \mathrm{Cl}_{7} \mathrm{O}_{4} \mathrm{P} \cdot 0.3$ EtOAc: C, 19.23; H, 2.33. Found: C, 19.15; H, 2.21. 
*Due to chiral center and prochirality there is four ${ }^{1} \mathrm{H}$ and two ${ }^{13} \mathrm{C}$ chemical shifts for $\mathrm{OCH}_{2}$-groups

Phosphoric acid 1-chloro-ethyl ester methyl ester phenyl ester 3d: $87 \%$ (without purification), white solid. Chromatography eluent $\mathrm{CH}_{2} \mathrm{Cl}_{2}$. Major isomer ca. 80\%: ${ }^{1} \mathrm{H} \mathrm{NMR}\left(\mathrm{CDCl}_{3}\right) \delta$ 7.380-7.325 $(2 \mathrm{H}, \mathrm{m}), 7.260-7.179(3 \mathrm{H}, \mathrm{m}), 6.328-6.271(1 \mathrm{H}, \mathrm{m}), 3.929\left(3 \mathrm{H}, \mathrm{d},{ }^{3} \mathrm{~J}_{\mathrm{HP}}=11.69 \mathrm{~Hz}\right), 1.796(3 \mathrm{H}, \mathrm{d}, \mathrm{J}=5.60$ $\mathrm{Hz}) ;{ }^{13} \mathrm{C} \mathrm{NMR}\left(\mathrm{CDCl}_{3}\right) \delta 150.13\left(\mathrm{~d},{ }^{2} \mathrm{~J}_{\mathrm{CP}}=7.0 \mathrm{~Hz}\right), 129.82,125.54,120.06\left(\mathrm{~d},{ }^{3} \mathrm{~J}_{\mathrm{CP}}=4.7 \mathrm{~Hz}\right), 86.08(\mathrm{~d}$, $\left.{ }^{2} \mathrm{~J}_{\mathrm{CP}}=6.2 \mathrm{~Hz}\right), 55.30\left(\mathrm{~d},{ }^{2} \mathrm{~J}_{\mathrm{CP}}=6.3 \mathrm{~Hz}\right), 27.34\left(\mathrm{~d},{ }^{3} \mathrm{~J}_{\mathrm{CP}}=8.6 \mathrm{~Hz}\right) ;{ }^{31} \mathrm{P} \mathrm{NMR}\left(\mathrm{CDCl}_{3}\right) \delta-6.94$; Minor isomer ca $20 \%$ : $\delta$ 7.380-7.325 $(2 \mathrm{H}, \mathrm{m}), 7.260 .7 .179(3 \mathrm{H}, \mathrm{m}), 6.342-6.280(1 \mathrm{H}, \mathrm{m}), 3.887(3 \mathrm{H}, \mathrm{d}$, $\left.{ }^{3} \mathrm{~J}_{\mathrm{HP}}=11.59 \mathrm{~Hz}\right), 1.888(3 \mathrm{H}, \mathrm{d}, \mathrm{J}=5.64 \mathrm{~Hz}) .{ }^{13} \mathrm{C} \mathrm{NMR}\left(\mathrm{CDCl}_{3}\right) \delta 150.13\left(\mathrm{~d},{ }^{2} \mathrm{~J}_{\mathrm{CP}}=7.0 \mathrm{~Hz}\right), 129.77$, $125.46,120.06\left(\mathrm{~d},{ }^{3} \mathrm{~J}_{\mathrm{CP}}=4.7 \mathrm{~Hz}\right), 85.99\left(\mathrm{~d},{ }^{2} \mathrm{~J}_{\mathrm{CP}}=7.2 \mathrm{~Hz}\right), 55.30\left(\mathrm{~d},{ }^{2} \mathrm{~J}_{\mathrm{CP}}=6.3 \mathrm{~Hz}\right), 27.55\left(\mathrm{~d},{ }^{3} \mathrm{~J}_{\mathrm{CP}}=8.6\right.$ $\mathrm{Hz}) ;{ }^{31} \mathrm{P}$ NMR $\left(\mathrm{CDCl}_{3}\right) \delta$-7.46. GC-MS m/z $251(\mathrm{M}+1), 215(\mathrm{M}-\mathrm{Cl})$. Anal. Calcd for $\mathrm{C}_{9} \mathrm{H}_{12} \mathrm{ClO}_{4} \mathrm{P}$ : C, 43.13; H, 4.83. Found: C, 43.21; H, 5.05.

Phosphoric acid 1-chloro-ethyl ester bis-(2,6-dimethyl-phenyl) ester 3e: $92 \%$ (without purification), colorless viscose oil. Chromatography eluent $\mathrm{CH}_{2} \mathrm{Cl}_{2} .{ }^{1} \mathrm{H} \mathrm{NMR}\left(\mathrm{CDCl}_{3}\right){ }^{1} \mathrm{H} \mathrm{NMR}\left(\mathrm{CDCl}_{3}\right)$ ઈ 7.050-6.969 (6H, m), $6.285\left(1 \mathrm{H}, \mathrm{dq}, \mathrm{J}=5.52 \mathrm{~Hz},{ }^{3} \mathrm{~J}_{\mathrm{HP}}=7.62 \mathrm{~Hz}\right), 2.403^{*}(6 \mathrm{H}, \mathrm{s}), 2.303^{*}(6 \mathrm{H}, \mathrm{s}), 1.694$ $(3 \mathrm{H}, \mathrm{dd}, \mathrm{J}=5.52 \mathrm{~Hz}) ;{ }^{13} \mathrm{C} \mathrm{NMR}\left(\mathrm{CDCl}_{3}\right) \delta 147.95\left(\mathrm{~d},{ }^{3} \mathrm{~J}_{\mathrm{CP}}=8.7 \mathrm{~Hz}\right), 147.76\left(\mathrm{~d},{ }^{3} \mathrm{~J}_{\mathrm{CP}}=8.7 \mathrm{~Hz}\right), 130.42$ $\left(\mathrm{d},{ }^{2} \mathrm{~J}_{\mathrm{CP}}=3.4 \mathrm{~Hz}\right), 130.34\left(\mathrm{~d},{ }^{2} \mathrm{~J}_{\mathrm{CP}}=3.4 \mathrm{~Hz}\right), 129.17\left(\mathrm{~d},{ }^{4} \mathrm{~J}_{\mathrm{CP}}=1.7 \mathrm{~Hz}\right), 129.09\left(\mathrm{~d},{ }^{4} \mathrm{~J}_{\mathrm{CP}}=1.7 \mathrm{~Hz}\right), 125.60$ $\left(\mathrm{d},{ }^{5} \mathrm{~J}_{\mathrm{CP}}=2.0 \mathrm{~Hz}\right), 125.55\left(\mathrm{~d},{ }^{5} \mathrm{~J}_{\mathrm{CP}}=2.0 \mathrm{~Hz}\right), 86.66\left(\mathrm{~d},{ }^{2} \mathrm{~J}_{\mathrm{CP}}=6.4 \mathrm{~Hz}\right), 27.22\left(\mathrm{~d},{ }^{3} \mathrm{~J}_{\mathrm{CP}}=8.1 \mathrm{~Hz}\right), 17.22$, 16.99; ${ }^{31} \mathrm{P} \mathrm{NMR}\left(\mathrm{CDCl}_{3}\right) \delta$-12.99. GC-MS m/z $369(\mathrm{M}+1), 333(\mathrm{M}-\mathrm{Cl})$. Anal. Calcd for $\mathrm{C}_{18} \mathrm{H}_{22} \mathrm{ClO}_{4} \mathrm{P}: \mathrm{C}, 58.62 ; \mathrm{H}, 6.01$. Found: C, 59.02; H, 6.27.

2-(1-Chloro-ethoxy)-[1,3,2]dioxaphosphinane 2-oxide 3f: $98 \%$ (without purification), colorless oil. Chromatography eluent hexane:EtOAc 1:4. ${ }^{1} \mathrm{H}$ NMR $\left(\mathrm{CDCl}_{3}\right){ }^{1} \mathrm{H}$ NMR $\left(\mathrm{CDCl}_{3}\right) \delta 6.309(1 \mathrm{H}, \mathrm{dq}$, $\left.\mathrm{J}=5.59 \mathrm{~Hz},{ }^{3} \mathrm{~J}_{\mathrm{HP}}=8.05 \mathrm{~Hz}\right), 4.560-4.399(4 \mathrm{H}, \mathrm{m}), 2.390-2.810(1 \mathrm{H}, \mathrm{m}), 1.879\left(3 \mathrm{H}, \mathrm{dd}, \mathrm{J}=5.59 \mathrm{~Hz},{ }^{4} \mathrm{~J}_{\mathrm{HP}}=\right.$ $0.85 \mathrm{~Hz}), 1.833-1.773(1 \mathrm{H}, \mathrm{m}) ;{ }^{13} \mathrm{C} \mathrm{NMR}\left(\mathrm{CDCl}_{3}\right) \delta 85.44\left(\mathrm{~d},{ }^{2} \mathrm{~J}_{\mathrm{CP}}=6.2 \mathrm{~Hz}\right), 69.63\left(\mathrm{~d},{ }^{2} \mathrm{~J}_{\mathrm{CP}}=7.4 \mathrm{~Hz}\right)$, $69.00\left(\mathrm{~d},{ }^{2} \mathrm{~J}_{\mathrm{CP}}=7.2 \mathrm{~Hz}\right), 27.74\left(\mathrm{~d},{ }^{3} \mathrm{~J}_{\mathrm{CP}}=7.7 \mathrm{~Hz}\right), 25.75\left(\mathrm{~d},{ }^{3} \mathrm{~J}_{\mathrm{CP}}=7.4 \mathrm{~Hz}\right) ;{ }^{31} \mathrm{P} \mathrm{NMR}\left(\mathrm{CDCl}_{3}\right) \delta-9.64$. 
GC-MS m/z $201(\mathrm{M}+1), 165(\mathrm{M}-\mathrm{Cl})$. Anal. Calcd for $\mathrm{C}_{5} \mathrm{H}_{10} \mathrm{ClO}_{4} \mathrm{P}: \mathrm{C}, 29.94 ; \mathrm{H}, 5.03$. Found: C, $30.06 ; \mathrm{H}, 5.15$.

Phosphoric acid 1-chloro-ethyl ester dibenzyl ester 3h: $68 \%$ (without purification), colorless viscose oil. ${ }^{22}{ }^{1} \mathrm{H}$ NMR $\left(\mathrm{CDCl}_{3}\right){ }^{1} \mathrm{H} \mathrm{NMR}\left(\mathrm{CDCl}_{3}\right) \delta$ 7.397-7.298 $(10 \mathrm{H}, \mathrm{m}), 6.150(1 \mathrm{H}, \mathrm{dq}, \mathrm{J}=5.49 \mathrm{~Hz}$, $\left.{ }^{3} \mathrm{~J}_{\mathrm{HP}}=7.58 \mathrm{~Hz}\right), 5.120-5.051 *(4 \mathrm{H}, \mathrm{m}), 1.782(3 \mathrm{H}, \mathrm{dd}, \mathrm{J}=5.49 \mathrm{~Hz}) ;{ }^{13} \mathrm{C} \mathrm{NMR}^{\#}\left(\mathrm{CDCl}_{3}\right) \delta 135.18,128.75$, 128.59, 128.28, 85.84, 69.91, 27.54; ${ }^{31} \mathrm{P} \mathrm{NMR}\left(\mathrm{CDCl}_{3}\right) \delta-1.55$.

*Due to chiral center and prochirality all four $\mathrm{OCH}_{2}$-signals have different chemical shifts. ${ }^{\text {\# }}$ Due to decomposition peaks are broad and $\mathrm{J}_{\mathrm{CP}}$-coupling constants can not be calculated.

Diethyl-phosphoramidic acid 1-chloro-ethyl ester methyl ester 3i: $91 \%$ (without purification), slightly yellow solid. Chromatography eluent $0.5 \% \mathrm{MeOH}$ in $\mathrm{CH}_{2} \mathrm{Cl}_{2}$. Major isomer ca. 60\%: ${ }^{1} \mathrm{H} \mathrm{NMR}$ $\left(\mathrm{CDCl}_{3}\right) \delta 6.247-6.189(1 \mathrm{H}, \mathrm{m}), 3.682\left(3 \mathrm{H}, \mathrm{d},{ }^{3} \mathrm{~J}_{\mathrm{HP}}=11.38 \mathrm{~Hz}\right), 3.178-3.021(4 \mathrm{H}, \mathrm{m}), 1.828(3 \mathrm{H}, \mathrm{dd}$, $\left.\mathrm{J}=5.69 \mathrm{~Hz},{ }^{4} \mathrm{~J}_{\mathrm{HP}}=0.72 \mathrm{~Hz}\right), 1.141(6 \mathrm{H}, \mathrm{t}, \mathrm{J}=7.14 \mathrm{~Hz}) ;{ }^{13} \mathrm{C} \mathrm{NMR}\left(\mathrm{CDCl}_{3}\right) \delta 85.04\left(\mathrm{~d},{ }^{2} \mathrm{~J}_{\mathrm{CP}}=5.7 \mathrm{~Hz}\right), 52.92$ $\left(\mathrm{d},{ }^{2} \mathrm{~J}_{\mathrm{CP}}=5.8 \mathrm{~Hz}\right), 39.90\left(\mathrm{~d},{ }^{2} \mathrm{~J}_{\mathrm{CP}}=4.8 \mathrm{~Hz}\right), 27.87\left(\mathrm{~d},{ }^{3} \mathrm{~J}_{\mathrm{CP}}=7.6 \mathrm{~Hz}\right), 14.04\left(\mathrm{~d},{ }^{3} \mathrm{~J}_{\mathrm{CP}}=2.2 \mathrm{~Hz}\right) ;{ }^{31} \mathrm{P} \mathrm{NMR}$ $\left(\mathrm{CDCl}_{3}\right) \delta$ 10.14. Minor isomer ca. 40\%: ${ }^{1} \mathrm{H}$ NMR $\left(\mathrm{CDCl}_{3}\right) \delta 6.247-6.189(1 \mathrm{H}, \mathrm{m}), 3.737(3 \mathrm{H}, \mathrm{d}$, $\left.{ }^{3} \mathrm{~J}_{\mathrm{HP}}=11.45 \mathrm{~Hz}\right), 3.178-3.021(4 \mathrm{H}, \mathrm{m}), 1.819\left(3 \mathrm{H}, \mathrm{dd}, \mathrm{J}=5.49 \mathrm{~Hz},{ }^{4} \mathrm{~J}_{\mathrm{HP}}=0.71 \mathrm{~Hz}\right), 1.116(6 \mathrm{H}, \mathrm{t}, \mathrm{J}=7.08$ $\mathrm{Hz}) ;{ }^{13} \mathrm{C} \mathrm{NMR}\left(\mathrm{CDCl}_{3}\right) \delta 85.30\left(\mathrm{~d},{ }^{2} \mathrm{~J}_{\mathrm{CP}}=7.0 \mathrm{~Hz}\right), 53.12\left(\mathrm{~d},{ }^{2} \mathrm{~J}_{\mathrm{CP}}=5.8 \mathrm{~Hz}\right), 39.70\left(\mathrm{~d},{ }^{2} \mathrm{~J}_{\mathrm{CP}}=4.4 \mathrm{~Hz}\right)$, $28.01\left(\mathrm{~d},{ }^{3} \mathrm{~J}_{\mathrm{CP}}=7.2 \mathrm{~Hz}\right), 14.06\left(\mathrm{~d},{ }^{3} \mathrm{~J}_{\mathrm{CP}}=2.2 \mathrm{~Hz}\right) ;{ }^{31} \mathrm{P} \mathrm{NMR}\left(\mathrm{CDCl}_{3}\right) \delta 10.34 . \mathrm{GC}-\mathrm{MS} \mathrm{m} / z 230(\mathrm{M}+1)$, 194 (M - Cl). Anal. Calcd for $\mathrm{C}_{7} \mathrm{H}_{17} \mathrm{ClNO}_{3} \mathrm{P}: \mathrm{C}, 29.94 ; \mathrm{H}, 5.03$. Found: $\mathrm{C}, 30.06 ; \mathrm{H}, 5.15$. 